\title{
Laser Irradiated Transient Thermography Inspection of Iron-Zinc Alloy Coatings on Steel Substrates
}

\author{
by L. Kehoe, P.V.Kelly and G.M. Crean
}

National Microelectronics Research Centre, Lee Maltings, Prospect Row, Cork, Ireland

\begin{abstract}
The potential of static Laser Irradiated Transient Thermography (LIIT) as a non-destructive testing (NDT) method for the characterisation of thin binary Fe-Zn alloy coatings on steel substrates is investigated. The ability of static pulsed LITT to non-destructively distinguish the presence of both underalloyed phases at the alloy coating surface, and overalloyed phases at the alloy coating-substrate interface is demonstrated. The sensitivity of static pulsed LITT to the thickness of a buried layer of $\Gamma$ phase Fe-Zn alloy is discussed.
\end{abstract}

\section{Introduction}

Transient thermography is an extension of the basic infrared imaging technique which since the early 1970's has been used as a surface inspection tool [1]. Some transient thermography methods use a flashiamp as a heating source, and are classified as Flash Lamp Transient Thermography (FLTT) or Pulsed Video Thermography (PVT) [2]. Where the heating source is a pulsed laser the technique may be referred to as Laser Irradiated Transient Thermography (LITT) or Pulsed PhotoThermal Radiometry (PPTR) [3]. If a very short laser pulse is used for heating, the technique is well suited to the characterisation of thin film material systems. LITT may be performed by scanning the laser and detector, measuring the radiometry signal as a function of position on the sample, or in static mode, in which case the entire radiometric transient is measured. In this paper the static pulsed LITT technique is used to characterise thin binary $\mathrm{Fe}-\mathrm{Zn}$ alloy coatings on steel substrates. Its ability to non-destructively distinguish the presence of different phases in the coating is demonstrated, and the sensitivity of static pulsed LITT to the thickness of a buried layer of $\Gamma$-phase Fe-Zn alloy is discussed.

\section{The Fe-Zn binary alloy}

Fe-Zn binary alloy coatings are utilised to improve the oxidation resistance of steels to harsh environmental conditions. The Fe- $\mathrm{Zn}$ phase diagram is shown in Figure 1 . While pure $\mathrm{Zn}$ coatings (i.e. the so-called $\eta$ phase of $\mathrm{Fe}-\mathrm{Zn}$ ) provide a degree of oxidation resistance, the $\partial_{1}$ phase $\mathrm{Fe}-\mathrm{Zn}$ alloy provides a superior resistance to the environment. The alloy coating process involves the annealing of the substrate after coating with a layer of zinc. Alloying takes place by diffusion of iron from the steel substrate into the zinc. The iron alloy fraction (iron content of the alloy) is therefore lower at the surface and increases with depth down to the coating-substrate interface. The $\partial_{1}$-phase is the most desirable alloy phase from the point of view of steel protective coating, while other phases of the $\mathrm{Fe}-\mathrm{Zn}$ binary alloy system ( $\eta$ (pure $\mathrm{Zn}$ ), $\zeta$ and $\Gamma$ ) suffer from brittleness or flaking in harsh environments. In the Fe-Zn alloy, variations in the iron alloy fraction through the coating must be confined within a small window $(8-13 \%)$ in order to produce the $\partial_{1}$-phase exclusively. Underalloying (emergence of the $\eta$ or $\xi$ phases) is most likely to occur near the surface of the coating. Overalloying (emergence of the $\Gamma$-phase) is most likely to occur near the coating-substrate interface, and will result in a thin buried layer of the mechanically poor $\Gamma$-phase interposed between the desired $\partial_{1}$-phase coating and the interface, impairing the coating performance. Ideally, the alloy fraction (\%Fe) will be greater than $8 \%$ at the surface, and less than $13 \%$ at the interface. A method of non-destructive detection and quantification of both surface underalloying and interfacial overalloying is required.

\section{Static pulsed LITT}

In transient thermography, the surface under inspection is heated by a laser pulse of short duration (typically between $5 \mathrm{~ns}$ and $50 \mu \mathrm{s}$ ) at a fluence well below the laser ablation threshold for the material. The transient blackbody radiation emission from the heated surface is then monitored with an infrared detector. Static pulsed LITT is therefore a form of pulsed photothermal radiometry [3]. The shape of the observed blackbody radiation transient, in the case of one-dimensional heat flow in the direction normal to the surface, is well known [4] and is related to the ability of the material system to dissipate a heat pulse, characterised by the thermal diffusivity $D$, and the optical absorption coefficients $\alpha$ and $\alpha^{\prime}$ of the material at the laser 
and blackbody radiation wavelengths respectively. The authors have previously [5] discussed certain difficulties with this formalism, including parameter correlation between the optical constants and the thermal diffusivity, the experimental difficulties in measuring $\alpha$ and $\alpha^{\prime}$ for many materials, and the inappropriateness of representing the optical absorption over a broad infrared spectrum (typically 8-12 $\mu \mathrm{m}$ ) by a single absorption coefficient. For the comparative inspection of materials whose optical absorption coefficient is large and approximately independent of spectral position in the visible to near infrared region (such as metals), differences in the shape of the front surface blackbody radiation transient from sample to sample of similarly absorbing materials are effectively determined by the thermal diffusivity of the material. However, where there is a marked difference in the surface optical properties, changes in the LITT transient shape may arise due to either changes in either the thermal diffusivity or the optical absorption coefficients. LITT transient shapes may also be influenced by any unusual properties of the material system, for example, by defects such as disbonds or buried layers. Static pulsed LITT may therefore be employed comparatively as a nondestructive test method to detect variations of material properties from a known norm, without a precise knowledge of the thermal diffusivity or optical absorption coefficients.

\section{Experimental methods}

A description of the laser flash radiometry instrument used in this work has already been published [5]. Figure 2 shows the configuration used for static pulsed LITT. A Nd:YAG laser pulse of $7 \mathrm{~ns}$ duration is employed to heat the test surface, and a Ge-filtered $\mathrm{HgCdTe}$ photovoltaic infrared detector of bandwidth $52 \mathrm{MHz}$ detects the blackbody radiation. The detector linearity with temperature was verified using a mechanically chopped calibrated blackbody source.

A standard set of iron-zinc ( $F e-Z n$ ) binary alloy coated steel substrates was inspected using LITT. Manufacturers' data on the Fe-Zn phases present in the coatings, and their layer thicknesses, determined using $X$-ray diffraction and coulometry, together with the coating mass per unit area and mean percentage of iron in the coating, are detailed below (tables 1 and 2).

\begin{tabular}{|l|l|l|l|l|l|l|}
\hline Sample & $\begin{array}{l}\text { Coating } \\
\text { mass per } \\
\text { unit area } \\
\left(\mathrm{g} . \mathrm{m}^{-2}\right)\end{array}$ & $\begin{array}{l}\text { Mean } \\
\% \mathrm{Fe} \\
\text { in coating }\end{array}$ & $\begin{array}{l}\text { X-ray diffraction defermination of Fe-Zn alloy phases } \\
\text { present (thickness of phase layer in } \mu \mathrm{m} \text { ) (as supplied by } \\
\text { manufacturer) }\end{array}$ \\
\hline Number & & & $\eta(\mu \mathrm{m})$ & $S(\mu \mathrm{m})$ & $\partial_{1}(\mu \mathrm{m})$ & $\Gamma(\mu \mathrm{m})$ \\
\hline 1 & 38.00 & 4.22 & $\mathrm{~L}$ & $\mathrm{~S}$ & 5.40 & ND \\
\hline 2 & 38.10 & 6.38 & $S-M$ & S-M & 6.70 & ND \\
\hline 3 & 39.40 & 8.88 & ND & T-S & 6.90 & 0.20 \\
\hline 4 & 42.90 & 12.31 & ND & T & 6.80 & 0.70 \\
\hline 5 & 42.40 & 13.14 & ND & T & 7.00 & 0.90 \\
\hline 6 & 43.30 & 13.91 & ND & T & 7.10 & 1.30 \\
\hline
\end{tabular}

Table 1. Coating mass per unit area, percentage $\mathrm{Fe}$ content; and $\mathrm{X}$-ray diffraction determination of the thickness of each phase layer present in the Fe- $\mathrm{Zn}$ alloy coatings. ( $N D=$ not detected, $L=$ large, $M=$ medium, $S=$ small, $T=$ trace.)

\begin{tabular}{|l|l|l|l|l|l|}
\hline Sample & $\begin{array}{c}\text { Surface } \\
\text { Phase }\end{array}$ & \multicolumn{4}{|c|}{ Thickness of Fe/Zn phaselayers present } \\
\hline $\begin{array}{c}\text { Scanning } \\
\text { Electron } \\
\text { Microscopy }\end{array}$ & Coulometry & Coulometry & Coulometry & Metallography \\
\hline Number & $\eta$ & $\eta(\mu \mathrm{m})$ & $\zeta(\mu \mathrm{m})$ & $\delta 1(\mu \mathrm{m})$ & $\Gamma(\mu \mathrm{m})$ \\
\hline 1 & $\eta$ & 0.42 & 2.05 & 3.07 & $<0.5$ \\
\hline 2 & $\eta / \zeta$ mixture & 0.11 & 0.61 & 4.57 & $<0.5$ \\
\hline 3 & $\delta 1$ & ND & ND & 5.07 & $<0.5$ \\
\hline 4 & $\delta 1$ & ND & ND & 4.52 & 1.00 \\
\hline 5 & $\delta 1$ & ND & ND & 4.52 & $1.0-1.5$ \\
\hline 6 & $\delta 1$ & ND & ND & 4.94 & $1.0-1.5$ \\
\hline
\end{tabular}

Table 2. Sample data measured by coulometry and metallography. ND = not detected. 
SEM microscopy data supplied by the manufacturer revealed the dominant phase present at the surface of the coatings, as documented in table 2. On visual inspection of the samples, a more highly reflecting surface was evident from those samples in which either the $\eta$ or $\zeta$ phases were dominant near the surface (i.e. for underalloyed samples).

\section{Results and Discussion}

In figure 3 the front surface radiometry transients acquired from samples 1,2 and 3 under similar irradiation and signal capture conditions are plotted. Sample 1, on which the dominant surface phase is $\eta$, is bright and highly reflective on visual inspection, compared to sample 2 on which the surface is a mixture of $\zeta$ and $\partial_{1}$ phases, and sample 3 , on which the surface is entirely $\partial_{1}$ phase. It therefore reflects a greater proportion of the incident laser pulse energy, and because its emissivity is reduced, gives a significantly lower infrared signal per degree of temperature excursion. For both reasons, the amplitude of the thermal transient of sample 1 is much smaller than that of sample 2 . Figure 4 shows the normalised transients for samples 1,2 and 3 , where both the aforementioned effects have been removed. In this case, the comparison is between the shape of the decay portion of the transients only, and a progressive slowing of the cooling portion of the transients is observed from sample 1 to sample 3 , as the percentage of iron in the coatings rises, and the coatings become uniformly $\partial_{1}$ phase. This sensitivity of the LITT transient shape to surface phase may arise either via differences in the thermal diffusivity of the different phases, or via differences in the optical absorption coefficient at either or both the laser and blackbody radiation wavelengths, because the surface layer absorbs the laser pulse and emits the detected blackbody signal. Parameter correlation between the optical absorption coefficient and the thermal diffusivity [5] does not allow the parameter which is the source of the variation in LITT transient shape to be distinguished.

Figure 5 shows the sensitivity of the LITT transient shape for samples 4,5 and 6 to the thickness of a buried $\Gamma$-phase layer of Fe-Zn varying in thickness between 0.7 and $1.3 \mu \mathrm{m}$ at a depth of approximately $7 \mu \mathrm{m}$ below a $\partial_{1}$-phase layer. The thicker $\Gamma$-phase layer appears to provide a better thermal flow between the $\partial_{1}$-phase coating and the steel substrate, resulting in a marginally faster surface cooling transient with increasing thickness of this $\Gamma$-phase, compared to the slower cooling transients observed with increasing $\partial_{1}$ phase thickness. These samples allow a good comparison of the $\Gamma$-phase layer effects because they all have very similar surface characteristics and $\partial_{1}$ phase thicknesses, and only differ in an increasing percentage of iron content in the alloy which correlates with the emergence of successively thicker $\Gamma$-phase layers at the interface with the underlaying steel substrate. No $\eta$ or $\zeta$ phases have been detected by either coulometry or X-Ray diffraction for these samples and SEM analysis shows the surface phase to be the $\partial_{\uparrow}$ phase.

The effects of the $\Gamma$-phase interface layer thickness are clearly second order effects compared to the major effects of $\eta$ and $\zeta$ phase layers at the surface, and are only perceptible in the absence of the larger effects. The sensitivity of LITT is a function of depth and of the laser pulse duration and the detector speed. Interfacial effects due to a buried layer are absent from the early portion of a thermal transient, and only manifest themselves when the heat pulse diffuses to that depth. The characteristic transit time $t$ for heat to diffuse to a depth $L$ within a bulk solid has been defined [4] as:

$$
t_{1}=\frac{L^{2}}{\pi^{2} D}
$$

An estimate of the delay before the effects of the $\Gamma$-phase interface layer could manifest themselves the transient shape made for a pure zinc (i.e. $\eta$-phase) coating $\left(D=4.18 \times 10^{-5}\right.$ ) of $7 \mu \mathrm{m}$ thickness (the approximate depth at which the $\Gamma$-phase interface layer is buried), using equation (1), gives a characteristic thermal transit time of $120 \mathrm{~ns}$ before the heat pulse diffuses to the $\Gamma$-phase interface layer. By contrast, effects due to an underalloyed $\eta$ or $\zeta$ phase layer at the surface would be expected to manifest themselves almost immediately at the start of the transient.

These effects are observed in the experimental data. In figure 4 , it is evident that the marked differences between the $\eta$ and $\partial_{1}$ phase coating surfaces emerge within $20 \mathrm{~ns}$ of the start of the cooling transient. In figure 5 , the effects of the buried $\Gamma$-phase interface layer are not evident in 
the first 100 ns of the transients, but emerge only as the heat pulse diffuses to the depth at which the $\Gamma$-phase interface layer is known to be buried. This is strong evidence to support a conclusion that these small second-order effects are indeed due to the buried effects of the $\Gamma$ phase interface layer, rather than other minor differences in the top layer of the coating. The differences in the transients, which are correlated with the thickness of this $\Gamma$-phase interface layer persist for several thousand nanoseconds via their effect on the transport of heat from the surface of the coating. In this case, the effect must be due to heat transport effects, as heat flow is the only physical phenomenon related to the transient shape which takes place at the depth of the buried layer. An apparently small improvement of heat flow from the surface with a thicker $\Gamma$-phase interface layer is observed.

The results demonstrate that both the amplitude of the transient and the shape of the cooling portion of the transient can be related to the surface phase present and to the coating thickness, providing a potential basis for the detection of underalloyed coating surfaces.

\section{Conclusions}

In conclusion, LITT is shown to be effective at detecting underalloyed and overalloyed Fe- $\mathrm{Zn}$ alloy coating surfaces. In the case of surface phase differences, the effect is seen in both the signal amplitude and the shape of the cooling transient, and is observed promptly. Parameter correlation does not allow the effect of surface phase on the transient shape to be specifically assigned to differences in the thermal diffusivity or the optical absorption function of the phases, but only to an indeterminate combination of both effects. More importantly, evidence has been found that LITT can distinguish small variations in the thickness of a buried $\Gamma$-phase layer, where it exists. In this case, the effect of the buried layer are delayed, and only appear in that portion of the LITT transient recorded after the heat pulse has diffused to the depth of the buried layer. These initial results form a basis for the development of a non-destructive quantitative test of the alloy fraction and gradient in binary alloys.

\section{Acknowledgements}

This work was partly funded by the Commission of the European Communities DGXII under the Brite/EuRam research program within project no. 4334 "Application of Transient Thermography to Advance Industrial Non-destructive testing" (ATTAIN).

\section{REFERENCES}

[1]. HOBBS (C.)- Transient Thermography. Sensor Review 12 (1992) 8-13

[2]. LAU (S.K.) , ALMOND (D.P.), and MILNE (J.M.)- A quantitative analysis of pulsed video thermography. NDT\&E International, 24 (1992) 195-202

[3]. TAM (A.C.) and SULLIVAN (B.)- Remote sensing applications of pulsed photothermal radiometry. Appl. Phys. Lett. 43 (1983) 333

[4]. LEUNG (W.L.) and TAM (A.C.)- Techniques of flash radiometry. J. Appl. Phys. 56 (1984) 153

[5]. KEHOE (L.), KELLY (P.V.), O'CONNOR (G.M.) O'REILLY (M.) and CREAN (G.M.)- A measurement methodology for laser-based thermal diffusivity measurement of advanced multichip module ceramic materials. IEEE Trans. Components, Packaging and Manufacturing Technology, Part A Vol 18, No. 4 p $773-780$ 


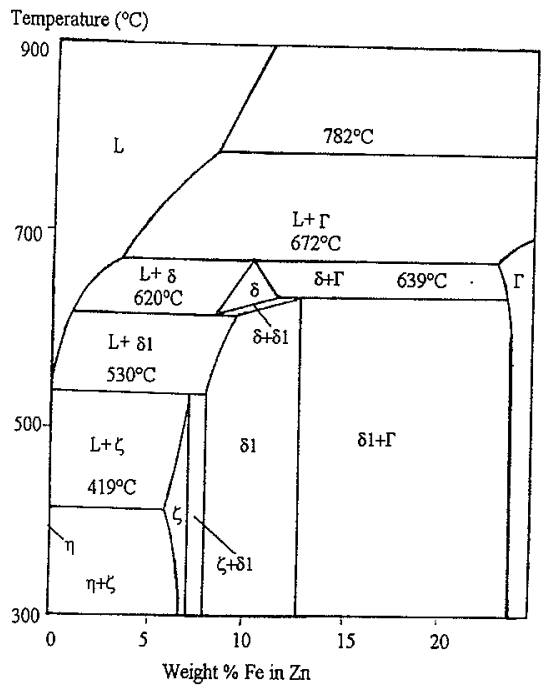

Figure 1: The Fe-Zn alloy phase diagram

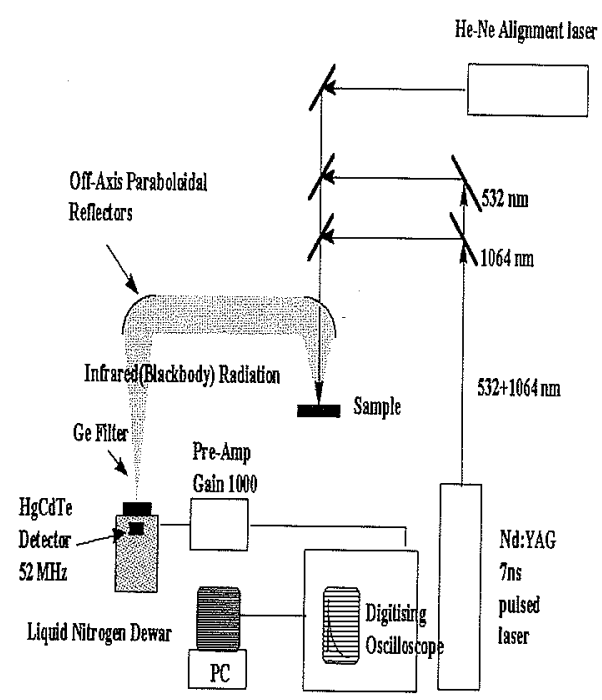

Figure 2: The static pulsed LITT instrument

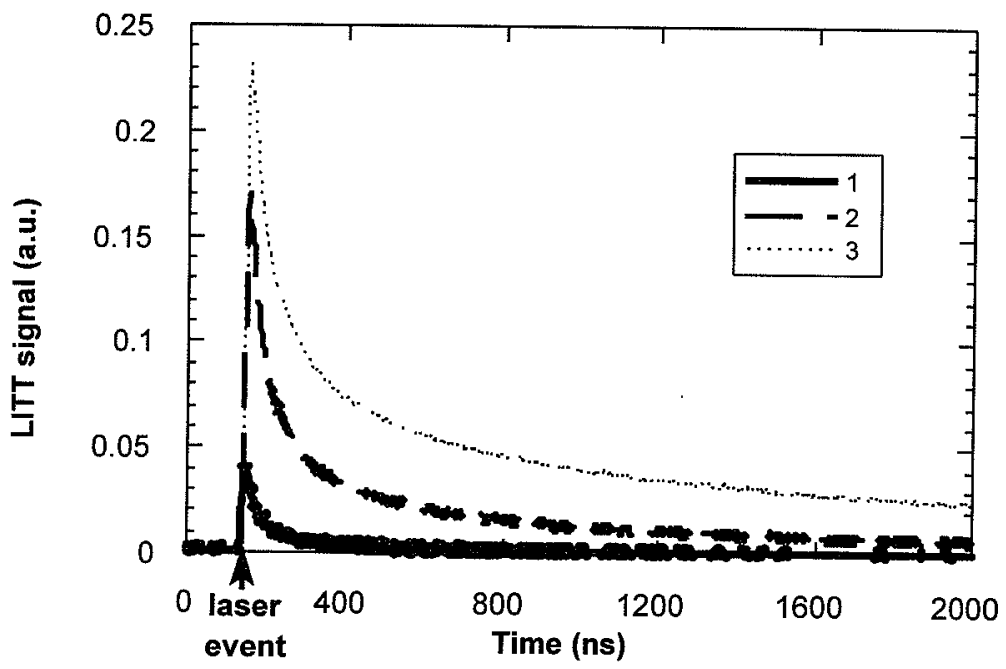

Figure 3. LITT signals from $\eta$ surface phase (sample 1), mixed $\zeta-\partial_{1}$ surface phase (sample 2) and $\partial_{1}$ surface phase (sample 3 ) Fe-Zn alloy coatings on steel. 


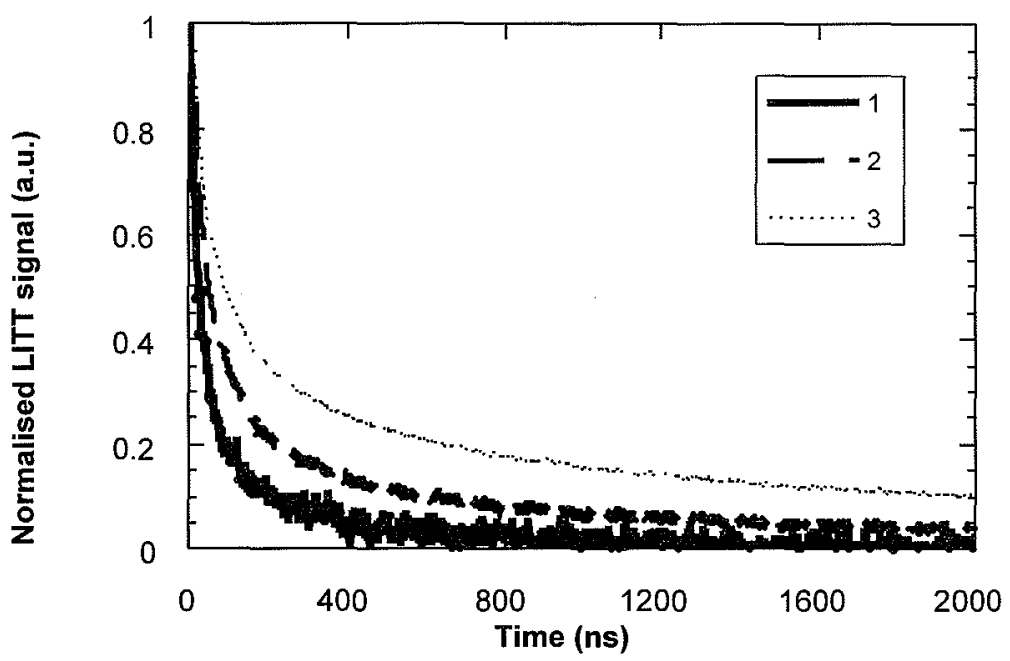

Figure 4: Normalised LITT transients from $\eta$ surface phase (sample 1), mixed $\zeta-\partial_{1}$ surface phase (sample 2) and $\partial_{1}$ surface phase (sample 3 ) Fe-Zn alloy coated steel.

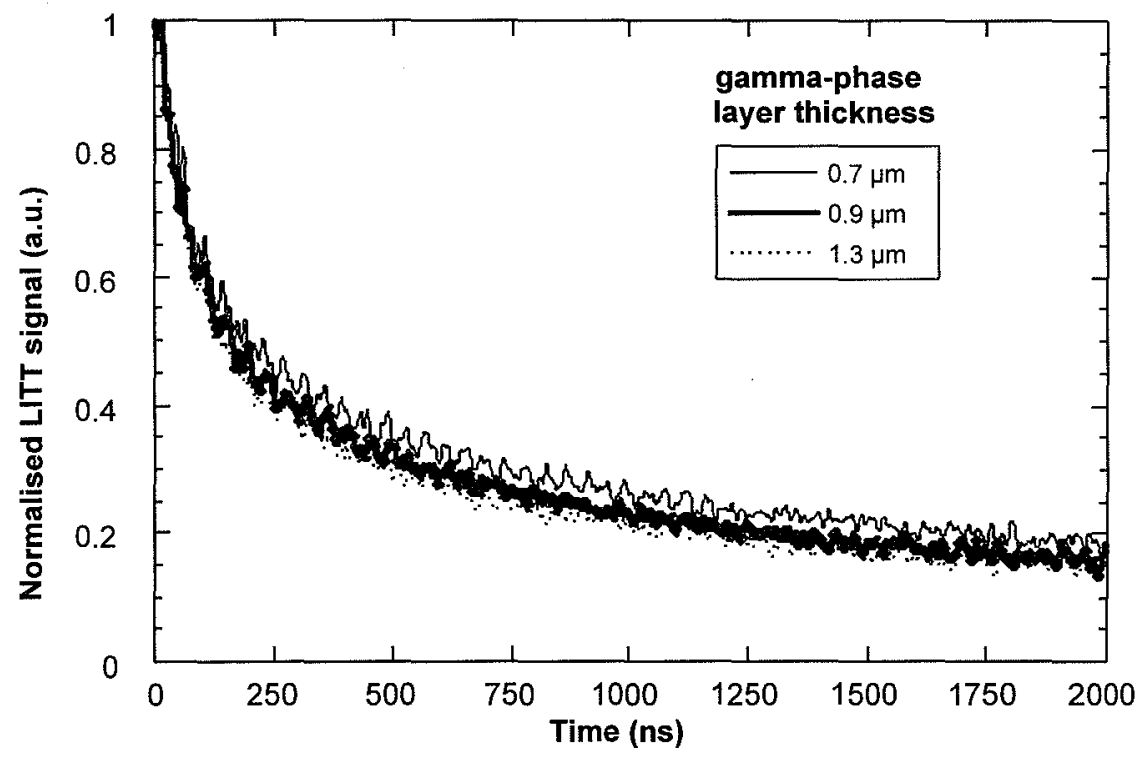

Figure 5: Normalised LITT transients from steel substrates with $\Gamma$-phase Fe-Zn layers of thickness $0.7 \mu \mathrm{m}, 0.9 \mu \mathrm{m}$ and $1.3 \mu \mathrm{m}$ below a $7 \mu \mathrm{m}$ thick $\partial_{1}$-phase layer. 